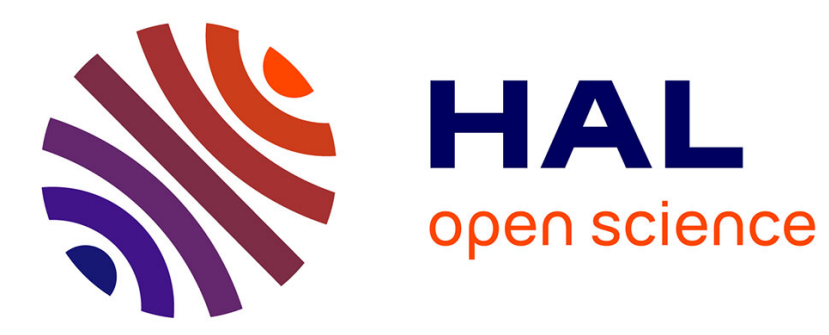

\title{
Local indeterminacy under dynamic efficiency two-sector overlapping generations economy
}

\author{
Carine Nourry, Alain Venditti
}

\section{To cite this version:}

Carine Nourry, Alain Venditti. Local indeterminacy under dynamic efficiency two-sector overlapping generations economy. 2009. halshs-00432270

\author{
HAL Id: halshs-00432270 \\ https://shs.hal.science/halshs-00432270 \\ Preprint submitted on 15 Nov 2009
}

HAL is a multi-disciplinary open access archive for the deposit and dissemination of scientific research documents, whether they are published or not. The documents may come from teaching and research institutions in France or abroad, or from public or private research centers.
L'archive ouverte pluridisciplinaire $\mathbf{H A L}$, est destinée au dépôt et à la diffusion de documents scientifiques de niveau recherche, publiés ou non, émanant des établissements d'enseignement et de recherche français ou étrangers, des laboratoires publics ou privés. 


\section{GREQAM}

Document de Travail

Groupement de Recherche en Economie Quantitative d'Aix-Marseille - UMR-CNRS 6579 Ecole des Hautes Etudes en Sciences Sociales Universités d'Aix-Marseille II et III

LOCAL INDETERMINACY UNDER DYNAMIC EFFICIENCY IN A TWO-SECTOR OVERLAPPING GENERATIONS ECONOMY

Carine NOURRY Alain VENDITTI

September 2009

$n^{\circ}$ 2009-27

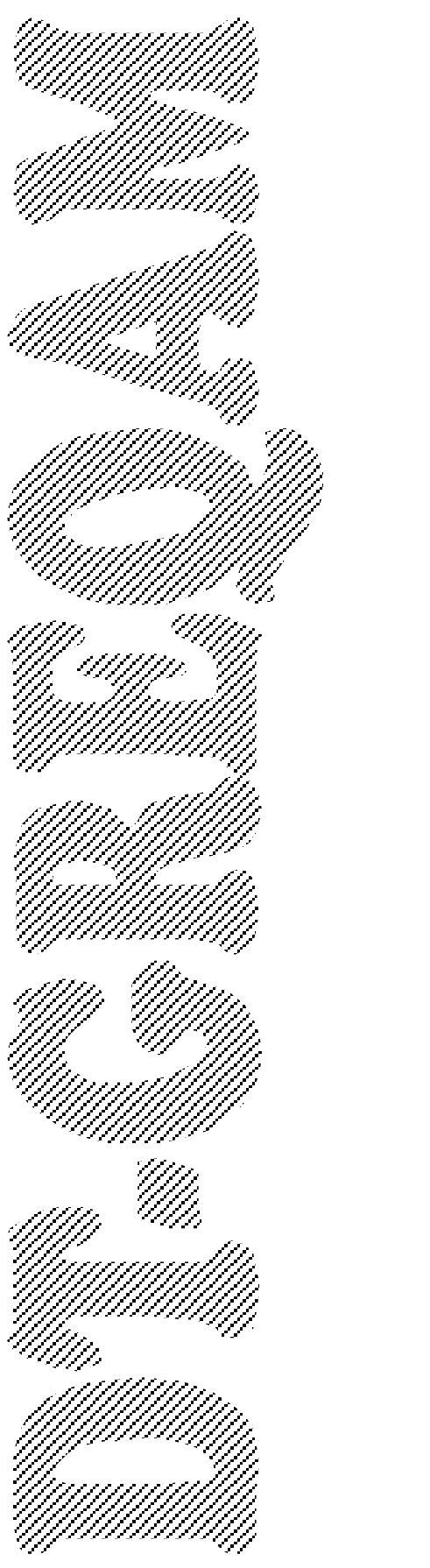




\title{
Local indeterminacy under dynamic efficiency in a two-sector overlapping generations economy*
}

\author{
Carine NOURRY ${ }^{\dagger}$ \\ Université de la Méditerranée and GREQAM \\ and \\ Alain VENDITTI ${ }^{\ddagger}$ \\ CNRS - GREQAM and EDHEC
}

\begin{abstract}
We consider a two-sector two-periods overlapping generations model with inelastic labor, consumption in both periods of life and homothetic CES preferences. Assuming gross substitutability and a capital intensive consumption good, we prove that when dynamic efficiency holds, local indeterminacy and sunspot fluctuations occur with low enough values for the sectoral elasticities of capital-labor substitution and we illustrate this finding within a standard example. This result shows that some fiscal policy rules can prevent the existence of business-cycle fluctuations in the economy by driving it to the optimal steady state as soon as it is announced, and thus shows that Reichlin's [9] influential conclusion is a robust property in a two-sector OLG economy.
\end{abstract}

Keywords: Two-sector OLG model, dynamic efficiency, gross substitutability in consumption, local indeterminacy, stabilization policy

Journal of Economic Literature Classification Numbers: C62, E32, O41.

${ }^{*}$ This work was supported by French National Research Agency Grant (ANR-08BLAN-0245-01). We would like to thank G. Bloise, J.P. Drugeon, R. Farmer, C. Ghiglino, J.M. Grandmont and P. Pintus for useful comments and suggestions.

${ }^{\dagger}$ E-mail: Carine.Nourry@univmed.fr

${ }^{\ddagger}$ E-mail: Alain.Venditti@univmed.fr 


\section{Introduction}

The existence of local indeterminacy and sunspot fluctuations under gross substitutability are well established facts whithin OLG economies. Considering an aggregate model with endogenous labor and consumption in the second period of life only, Reichlin [9] has shown that Hopf cycles and thus local indeterminacy arise with a unique Pareto optimal steady state when the technology is Leontief. This conclusion has had a strong echo in the literature as it implies that the introduction of a public policy based on taxes and transfers could at the same time stabilize the economy and reach the Pareto optimal steady state along which all generations get an equal level of utility.

However, Cazzavillan and Pintus [3] have recently proved that the coexistence of local indeterminacy and dynamic efficiency is not robust to the consideration of any positive elasticity of capital-labor substitution. Indeed, apart from the very special case of Leontief technology, the steady state is always characterized by an over-accumulation of capital when local indeterminacy holds, ${ }^{1}$ and policies targeting the steady state allocation generically leave room for welfare losses associated with productive inefficiency.

As initially shown in Galor [6], local indeterminacy also arises in twosector OLG economies under gross substitutability. However, the Pareto optimality of the equilibrium has not been precisely discussed in such a framework. On the one hand, Reichlin [10] proves local indeterminacy of a dynamically inefficient steady state through the existence of periodic, quasiperiodic and chaotic dynamics under the assumption of Leontief technologies. On the other hand, Drugeon et al. [5] show that any dynamically efficient equilibrium path is locally determinate if the sectoral elasticities of capital-labor substitution are large enough.

Our main objective in this paper is to show that Reichlin's [9] result is a robust property in a two-sector OLG economy. Assuming gross substitutability and a capital intensive consumption good sector, we prove indeed that local indeterminacy can occur under dynamic efficiency when intermediary values for the elasticity of intertemporal substitution in consumption and low enough but positive sectoral elasticities of capital-labor substitution are considered. This conclusion shows that contrary to the aggregate framework, in two-sector OLG economies with capital-labor substitutability, some fiscal policy rules can eliminate business-cycle fluctuations in the economy

\footnotetext{
${ }^{1}$ See also Cazzavillan and Pintus [2] for an extension of this result to a model with positive first-period consumption.
} 
by driving it to an optimal steady state as soon as it is announced.

\section{The model}

\subsection{Production}

The economy consists in one consumption good $y_{0}$ and one capital good $y$ produced from capital and labor through standard constant returns to scale technologies:

$$
y_{0}=f^{0}\left(k^{0}, l^{0}\right), \quad y=f^{1}\left(k^{1}, l^{1}\right) \text { with } k^{0}+k^{1} \leq k \text { and } l^{0}+l^{1} \leq \ell
$$

$k$ being the total stock of capital and $\ell$ the total amount of labor.

Assumption 1. Each production function $f^{i}: \mathbb{R}_{+}^{2} \rightarrow \mathbb{R}_{+}, i=0,1$, is $C^{2}$, increasing in each argument, concave, homogeneous of degree one and such that for any $x>0, f_{1}^{i}(0, x)=f_{2}^{i}(x, 0)=+\infty, f_{1}^{i}(+\infty, x)=f_{2}^{i}(x,+\infty)=0$.

As $y \leq f^{1}(k, \ell)$, Assumption 1 implies that there exists $\bar{k}(\ell)>0$ solution of $k-f^{1}(k, \ell)=0$ such that $f^{1}(k, \ell)>k$ when $k<\bar{k}(\ell)$, while $f^{1}(k, \ell)<k$ when $k>\bar{k}(\ell)$. The set of admissible 3 -uples $(k, y, \ell)$ is thus defined as

$$
\tilde{\mathcal{K}}=\left\{(k, y, \ell) \in \mathbb{R}_{+}^{3} \mid 0<\ell, 0 \leq k \leq \bar{k}(\ell), 0 \leq y \leq f^{1}(k, \ell)\right\}
$$

For any $(k, y, \ell)$, profit maximization in the representative firm of each sector is equivalent to solving the following problem of optimal allocation of productive factors between the two sectors:

$$
\begin{aligned}
T(k, y, \ell)=\max _{k^{0}, k^{1}, l^{0}, l^{1}} & f^{0}\left(k^{0}, l^{0}\right) \\
\text { s.t. } & y \leq f^{1}\left(k^{1}, l^{1}\right) \\
& k^{0}+k^{1} \leq k \\
& l^{0}+l^{1} \leq \ell \\
& k^{0}, k^{1}, l^{0}, l^{1} \geq 0
\end{aligned}
$$

The social production function $T(k, y, \ell)$ gives the maximal output of the consumption good along interior temporary equilibria $(k, y, \ell) \in \tilde{\mathcal{K}}$. Under Assumption $1, T(k, y, \ell)$ is homogeneous of degree one, concave and $C^{2}$ over $\tilde{\mathcal{K}}^{2}{ }^{2}$ Denoting $w$ the wage rate, $r$ the gross rental rate of capital and $p$ the price of investment good, all in terms of the price of the consumption good, we derive from the envelope theorem that

\footnotetext{
${ }^{2}$ See Benhabib and Nishimura [1].
} 


$$
r=T_{1}(k, y, \ell), \quad p=-T_{2}(k, y, \ell), \quad w=T_{3}(k, y, \ell)
$$

The share of capital in total income is then given by

$$
s(k, y, \ell)=\frac{r k}{T(k, y, \ell)+p y} \in(0,1)
$$

\subsection{Consumption and savings}

In each period $t, N_{t}$ agents are born, and they live for two periods. In their first period of life (when young), the agents are endowed with one unit of labor that they supply inelastically to firms. Their income results from the real wage and is allocated between current consumption and savings which are invested in the firms. In their second period of life (when old), they are retired and their income resulting from the return on the savings is entirely consumed. The utility function of a representative agent, defined over his consumption bundle ( $c_{t}$, when he is young, and $d_{t+1}$, when he is old), is

$$
u\left(c_{t}, d_{t+1}\right)=\left[c_{t}^{1-1 / \gamma}+\delta\left(d_{t+1} / B\right)^{1-1 / \gamma}\right]^{\gamma /(\gamma-1)}
$$

with $\delta \in(0,1)$ the discount factor, $\gamma>0$ the elasticity of intertemporal substitution in consumption and $B>0$ a scaling parameter.

Each agent is assumed to have $1+n>0$ children so that $N_{t+1}=$ $(1+n) N_{t}$. Under perfect foresight, and considering the wage rate $w_{t}$ and the gross rate of return $R_{t+1}$ as given, a young agent maximizes his utility function over his life-cycle as follows:

$$
\begin{array}{rl}
\max _{c_{t}, d_{t+1}, \phi_{t}} & u\left(c_{t}, d_{t+1}\right) \\
\text { s.t. } & w_{t}=c_{t}+\phi_{t} \\
& R_{t+1} \phi_{t}=d_{t+1} \\
& c_{t}, d_{t+1}, \phi_{t} \geq 0
\end{array}
$$

Solving the first order conditions gives:

$$
c_{t}=\frac{w_{t}}{1+\delta^{\gamma}\left(R_{t+1} / B\right)^{\gamma-1}} \equiv \alpha\left(R_{t+1} / B\right) w_{t}
$$

with $\alpha(R / B) \in(0,1)$ the share of first period consumption over the wage income. Under gross substitutability, $\gamma>1$ and the saving function $\phi_{t}=$ $\left(1-\alpha\left(R_{t+1} / B\right)\right) w_{t}$ is increasing in $R$.

\subsection{Perfect-foresight competitive equilibrium}

Total labor is given by the number $N_{t}$ of young households, i.e., $\ell_{t}=N_{t}$, and is increasing at rate $n$, i.e., $\ell_{t+1}=(1+n) \ell_{t}$. We also assume complete depreciation of capital within one period. 
Definition 1. A sequence $\left\{k_{t}, y_{t}, \ell_{t}, c_{t}, d_{t}, r_{t}, w_{t}, p_{t}\right\}_{t=0}^{\infty}$, with $\left(k_{0}, \ell_{0}\right)=$ $\left(\hat{k}_{0}, \hat{\ell}_{0}\right)$ given, is a perfect-foresight competitive equilibrium if:

i) $c_{t}=\alpha\left(R_{t+1} / B\right) w_{t}$;

ii) $\ell_{t}\left(1-\alpha\left(R_{t+1} / B\right)\right) w_{t}=p_{t} y_{t}$;

iii) $y_{t}=k_{t+1}$

iv) $\ell_{t+1}=(1+n) \ell_{t}$

v) $\ell_{t}\left[c_{t}+d_{t} /(1+n)\right]=T\left(k_{t}, y_{t}, \ell_{t}\right)$

vi) $\left(r_{t}, w_{t}, p_{t}\right)$ is given by (4);

vii) $R_{t+1}=r_{t+1} / p_{t}$.

Let $\kappa=k / \ell$ and $\bar{\kappa}$ be the solution of $\kappa-f^{1}(\kappa, 1)=0$. The set of admissible paths given by $(2)$ can be redefined as follows

$$
\mathcal{K}=\left\{\left(\kappa_{t}, \kappa_{t+1}\right) \in \mathbb{R}_{+}^{2} \mid 0 \leq \kappa_{t} \leq \bar{\kappa}, 0 \leq \kappa_{t+1} \leq f^{1}\left(\kappa_{t}, 1\right) /(1+n)\right\}
$$

A perfect-foresight competitive equilibrium then satisfies

$$
(1+n) \kappa_{t+1}+\frac{T_{3}\left(\kappa_{t},(1+n) \kappa_{t+1}, 1\right)}{T_{2}\left(\kappa_{t},(1+n) \kappa_{t+1}, 1\right)}\left[1-\alpha\left(-\frac{T_{1}\left(\kappa_{t+1},(1+n) \kappa_{t+2}, 1\right)}{T_{2}\left(\kappa_{t},(1+n) \kappa_{t+1}, 1\right) B}\right)\right]=0
$$

with $\alpha(R / B)$ given by $(7),\left(\kappa_{t}, \kappa_{t+1}\right) \in \mathcal{K}$ and $\kappa_{0}=\hat{\kappa}_{0}=\hat{k}_{0} / \hat{\ell}_{0}$ given.

\section{$3 \quad$ Steady state and dynamic efficiency}

\subsection{A normalized steady state}

A steady state is defined as $\kappa_{t}=\kappa^{*}$ for all $t$ with $\kappa^{*}$ solution of

$$
\alpha\left(-\frac{T_{1}(\kappa,(1+n) \kappa, 1)}{T_{2}(\kappa,(1+n) \kappa, 1) B}\right)=1+(1+n) \frac{\kappa T_{2}(\kappa,(1+n) \kappa, 1)}{T_{3}(\kappa,(1+n) \kappa, 1)} \equiv \Phi_{\kappa} \in(0,1)
$$

We consider a family of economies parameterized by $\gamma \neq 1$. We follow the same procedure as in Drugeon et al. [5]: we use the scaling parameter $B$ to ensure the existence of a normalized steady state (NSS) $\kappa^{*} \in(0, \bar{\kappa})$ which remains invariant as $\gamma$ is varied. From (7) and (10) we get:

Proposition 1. Under Assumption 1, let $\gamma \neq 1$ and $\kappa^{*} \in(0, \bar{\kappa})$. Then there exists a unique value $B\left(\kappa^{*}\right)>0$ as given by

$$
B\left(\kappa^{*}\right)=-\frac{T_{1}\left(\kappa^{*},(1+n) \kappa^{*}, 1\right)}{T_{2}\left(\kappa^{*},(1+n) \kappa^{*}, 1\right)}\left(\frac{-(1+n) \kappa^{*} T_{2}\left(\kappa^{*},(1+n) \kappa^{*}, 1\right)}{\delta^{\gamma}\left[T_{3}\left(\kappa^{*},(1+n) \kappa^{*}, 1\right)+(1+n) \kappa^{*} T_{2}\left(\kappa^{*},(1+n) \kappa^{*}, 1\right)\right]}\right)^{\frac{1}{1-\gamma}}
$$

such that $\kappa^{*}$ is a steady state if and only if $B=B\left(\kappa^{*}\right)$.

Proof: See Appendix 5.1.

In the rest of the paper we assume that $B=B\left(\kappa^{*}\right)$ so that $s=s\left(\kappa^{*}, \kappa^{*}, 1\right)$ and $\alpha=\alpha\left(R^{*} / B\left(\kappa^{*}\right)\right)=\Phi_{\kappa^{*}}$ remain constant as $\gamma$ is made to vary. 


\subsection{Dynamic efficiency}

From Definition 1 and the homogeneity of $T(k, y, \ell)$, considering that $\kappa^{*} T_{2} / T_{3}=\left(T_{2} / T_{1}\right)\left(\kappa^{*} T_{1} / T_{3}\right)=-s / R^{*}(1-s)$, we derive the stationary gross rate of return along the NSS:

$$
R^{*}=\frac{(1+n) s}{(1-\alpha)(1-s)}
$$

Under-accumulation of capital is obtained if and only if $R^{*}>1+n$. As shown in Drugeon et al. [5] we have:

Proposition 2. Under Assumption 1, let $\gamma \neq 1$ and $\underline{\alpha}=1-s /(1-s)$. Then:

i) the NSS is characterized by an under-accumulation of capital if and only if $\alpha \geq \underline{\alpha}$;

ii) an intertemporal competitive equilibrium converging towards the NSS is dynamically efficient if $\alpha \in(\underline{\alpha}, 1)$ and dynamically inefficient if $\alpha \in(0, \underline{\alpha})$.

If the labor income is relatively larger than the capital income $(s<1 / 2), \underline{\alpha}>$ 0 and young agents receive enough wage resources to provide a large amount of savings. But over-accumulation of capital can be avoided provided the share of first period consumption over the wage income is large enough, i.e. the agent does not save too much.

\section{Local indeterminacy under dynamic efficiency}

Let us introduce the relative capital intensity difference across sectors

$$
b \equiv\left(k^{1} / l^{1}-k^{0} / l^{0}\right) l^{1} / y
$$

and the elasticity of the rental rate of capital

$$
\varepsilon_{r k}=-T_{11}\left(\kappa^{*},(1+n) \kappa^{*}, 1\right) \kappa^{*} / T_{1}\left(\kappa^{*},(1+n) \kappa^{*}, 1\right)
$$

evaluated at the NSS. From now on we consider a positive value for $\underline{\alpha}=1-$ $s /(1-s)$, we assume gross substitutability, a capital intensive consumption good, ${ }^{3}$ and we consider dynamically efficient paths:

Assumption 2. $s \in(0,1 / 2), \gamma>1, b<0$ and $\alpha \geq \underline{\alpha}$.

We then prove that contrary to the aggregate OLG model, local indeterminacy arises under dynamic efficiency in a two-sector model with positive capital-labor substitution.

\footnotetext{
${ }^{3}$ When $\gamma>1$, this is a necessary condition for local indeterminacy (See Galor [6]).
} 
Theorem 1. Under Assumptions 1-2, let $\bar{b}=-1 /(1+n)$ and $\underline{b}=-(1-$ $\alpha) /(1+n) \alpha$. Then there exist $\underline{\varepsilon}_{r k}>0, \underline{\gamma}>1$ and $\bar{\gamma}>\underline{\gamma}$ such that the NSS is locally indeterminate if and only if $s \in(1 / 3,1 / 2), \alpha \in(\underline{\alpha}, 1 / 2), b \in(\underline{b}, \bar{b})$, $\varepsilon_{r k}>\underline{\varepsilon}_{r k}$ and $\gamma \in(\underline{\gamma}, \bar{\gamma})$.

Proof: See Appendix 5.2.

Theorem 1 is based on a large elasticity $\varepsilon_{r k}$. This restriction can be interpreted through the aggregate elasticity of capital-labor substitution $\Sigma$ which is linked to $\varepsilon_{r k}$ as follows (see Drugeon [4]):

$$
\Sigma=\frac{y_{0}+p y}{p y k y_{0}}\left(p y k^{0} l^{0} \sigma_{0}+y_{0} k^{1} l^{1} \sigma_{1}\right), \quad \varepsilon_{r k}=\left(l^{0} / y_{0}\right)^{2} \frac{w\left(y_{0}+p y\right)}{\Sigma}
$$

with $\sigma_{0}$ and $\sigma_{1}$ the sectoral elasticities of input substitution. Local indeterminacy under dynamic efficiency then requires a low enough aggregate elasticity $\Sigma$, i.e. low enough but still positive sectoral elasticities. Note also that the consumption good sector has to be sufficiently capital intensive. If $b$ is too close to 0 , i.e. if the model is close to the aggregate formulation, we get the same conclusion as Cazzavillan and Pintus [2, 3]: local indeterminacy under dynamic efficiency can never arise with any positive elasticity of capital-labor substitution.

Remark 1: Note that $\bar{\gamma}$ is generically a flip bifurcation value giving rise to period-two cycles which are locally indeterminate (or unstable) in a right (or left) neighborhood of $\bar{\gamma}$, while $\underline{\gamma}$ is generically a transcritical bifurcation value leading to the existence of a second steady state which is locally unstable (saddle-point stable) in a right (left) neighborhood of $\gamma$ (see Figure 1 in Appendix 5.2).

Through the NSS, the conditions on $\alpha, s, b$ and $\varepsilon_{r k}$ in Theorem 1 are based on joint restrictions of the technologies. We need therefore to show that they can be satisfied with standard production functions. Consider the following CES formulations:

$$
\begin{aligned}
& f^{0}\left(k^{0}, l^{0}\right)=\left[\chi\left(k^{0}\right)^{-\varsigma}+(1-\chi)\left(l^{0}\right)^{-\varsigma}\right]^{-\frac{1}{\varsigma}} \\
& f^{1}\left(k^{1}, l^{1}\right)=\left[\frac{1}{2}\left(\frac{k^{1}}{\eta}\right)^{-\rho}+{\frac{\left(l^{1}\right)}{2}}^{-\rho}\right]^{-\frac{1}{\rho}}
\end{aligned}
$$

with $\chi \in(0,1), \varsigma, \rho>-1$ and $\eta>0$. The sectoral elasticities of capital-labor substitution are given by $\sigma_{0}=1 /(1+\varsigma)$ and $\sigma_{1}=1 /(1+\rho)$. We assume for simplicity a constant population, i.e. $n=0$. Note that when $\sigma_{1}=0$ the investment good technology becomes Leontief as $\lim _{\rho \rightarrow+\infty} f^{1}\left(k^{1}, l^{1}\right)=$ 
$\min \left\{k^{1} / \eta, l^{1}\right\}$. Assume in a first step that $\sigma_{1}=0$. As $y=k^{1} / \eta=l^{1}$, we get

$$
T^{0}(k, y, \ell)=\left[\chi(k-\eta y)^{-\varsigma}+(1-\chi)(\ell-y)^{-\varsigma}\right]^{-1 / \varsigma}
$$

Following Proposition 1, we consider a NSS $\kappa=\kappa^{*} \in(0,1)$ and we compute

$$
\begin{gathered}
\alpha^{0}=\frac{(1-\chi)(1-\eta)^{1+\varsigma} \kappa^{* \varsigma}\left(1-\kappa^{*}\right)-\eta \chi\left(1-\kappa^{*}\right)^{1+\varsigma}}{(1-\chi)(1-\eta)^{1+\varsigma} \kappa^{* \varsigma}}, s^{0}=\frac{\chi\left(1-\kappa^{*}\right)^{1+\varsigma}}{(1-\chi)(1-\eta)^{1+\varsigma} \kappa^{* \varsigma}} \\
\underline{\alpha}^{0}=\frac{(1-\chi)(1-\eta)^{1+\varsigma} \kappa^{* \varsigma}-\chi\left(1-\kappa^{*}\right)^{1+\varsigma}}{(1-\chi)(1-\eta)^{1+\varsigma} \kappa^{* \varsigma}}, b^{0}=\frac{\eta-\kappa^{*}}{1-\kappa^{*}} \text { and } \varepsilon_{r k}^{0}=\frac{(1+\varsigma)(1-\chi) \kappa^{* \varsigma}}{(1-\eta)\left[\chi\left(\frac{1-\kappa^{*}}{\kappa^{*}(1-\eta)}\right)^{\varsigma}+1-\chi\right]}
\end{gathered}
$$

together with the bounds $\bar{b}=-1$,

$$
\begin{aligned}
\underline{b}^{0} & =-\frac{(1-\chi)(1-\eta)^{1+\varsigma} \kappa^{* 1+\varsigma}+\eta \chi\left(1-\kappa^{*}\right)^{1+\varsigma}}{(1-\chi)(1-\eta)^{1+\varsigma} \kappa^{*}\left(1-\kappa^{*}\right)-\eta \chi\left(1-\kappa^{*}\right)^{1+\varsigma}}, \\
\underline{\varepsilon}_{r k}^{0} & =\frac{\left(\kappa^{*}-\eta\right)\left(1-\kappa^{*}\right)(1-\chi)}{\left(2 \kappa^{*}-1-\eta\right)(1-\eta) \eta \chi} \frac{\chi(1+\eta)+(1-\chi)\left(\frac{\kappa^{*}(1-\eta)}{1-\kappa^{*}}\right)^{1+\varsigma}}{\chi\left(\frac{1-\kappa^{*}}{\kappa^{*}(1-\eta)}\right)^{\varsigma}+1-\chi}
\end{aligned}
$$

In a first step we derive that if $\kappa^{*} \in(1 / 2,1), \chi \in\left(\underline{\chi}_{1}^{0}, \bar{\chi}^{0}\right)$ with

$$
\underline{\chi}_{1}^{0}=\frac{(1-\eta)^{\varsigma} \kappa^{* 1+\varsigma}}{\left(1-\kappa^{*}\right)^{1+\varsigma}+(1-\eta)^{\varsigma} \kappa^{* 1+\varsigma}}, \quad \bar{\chi}^{0}=\frac{(1-\eta)^{1+\varsigma} \kappa^{* \varsigma}}{\left(1-\kappa^{*}\right)^{1+\varsigma}+(1-\eta)^{1+\varsigma} \kappa^{* \varsigma}}
$$

and $\eta<\bar{\eta}^{0}=\min \left\{1-\kappa^{*}, 2 \kappa^{*}-1\right\}$, then $s^{0} \in(1 / 3,1 / 2), \alpha^{0} \in\left(\underline{\alpha}^{0}, 1 / 2\right)$ and $b^{0} \in\left(\underline{b}^{0},-1\right)$. In a second step, we derive that if $\varsigma>\underline{\varsigma}^{0}$ with

$$
\underline{\varsigma}^{0}=\frac{\kappa^{*}\left[1-\eta(1-\eta)-\kappa^{* 2}\right]}{\left(2 \kappa^{*}-1-\eta\right) \eta}
$$

then there exists $\underline{\chi}_{2}^{0} \in(0, \bar{\chi})$ given by

$$
\underline{\chi}_{2}^{0}=\frac{\left(\kappa^{*}-\eta\right)\left(1-\kappa^{*}\right)\left(\frac{\kappa^{*}(1-\eta)}{1-\kappa^{*}}\right)^{1+\varsigma}}{\varsigma\left(2 \kappa^{*}-1-\eta\right) \eta-\kappa^{*}\left[1+\eta^{2}-\kappa^{*}(1+\eta)\right]+\left(\kappa^{*}-\eta\right)\left(1-\kappa^{*}\right)\left(\frac{\kappa^{*}(1-\eta)}{1-\kappa^{*}}\right)^{1+\varsigma}}
$$

such that $\varepsilon_{r k}>\underline{\varepsilon}_{r k}^{0}$ when $\chi>\underline{\chi}_{2}^{0}$. Denoting $\underline{\chi}^{0}=\max \left\{\underline{\chi}_{1}^{0}, \underline{\chi}_{2}^{0}\right\}$, we have then proved:

Lemma 1. Let the production functions be given by (15) with $\sigma_{1}=0$. If $\kappa^{*} \in(1 / 2,1), \eta<\bar{\eta}^{0}, \varsigma>\underline{\varsigma}^{0}$ and $\chi \in\left(\chi^{0}, \bar{\chi}^{0}\right)$, there exist $\gamma^{0}>1$ and $\bar{\gamma}^{0}>\underline{\gamma}^{0}$ such that the NSS is locally indeterminate for any $\left.\gamma \in \overline{(}^{0}, \bar{\gamma}^{0}\right)$.

Assume now that $\sigma_{1}>0$. The social production function derived as the value function of program (3) is parameterized by $\sigma_{1}$, namely $T^{\sigma_{1}}(k, y, \ell)$. Considering the same NSS $\kappa=\kappa^{*} \in(0,1)$, we can similarly compute $\alpha^{\sigma_{1}}$, $s^{\sigma_{1}}, \underline{\alpha}^{\sigma_{1}}, b^{\sigma_{1}}, \varepsilon_{r k}^{\sigma_{1}}, \underline{b}^{\sigma_{1}}$ and $\underline{\varepsilon}_{r k}^{\sigma_{1}}$. We have the following property:

Lemma 2. The social production function $T^{\sigma_{1}}(k, y, \ell)$ is continuous in $\left(k, y, \ell, \sigma_{1}\right)$, and as $\sigma_{1} \rightarrow 0$, converges to $T^{0}(k, y, \ell)$ uniformly in $(k, y, \ell)$, i.e. for any $\epsilon>0$, there is $\xi>0$ such that if $0<\sigma_{1}<\xi, \mid T^{\sigma_{1}}(k, y, \ell)-$ $T^{0}(k, y, \ell) \mid<\epsilon$ for any $(k, y, \ell) \in \tilde{\mathcal{K}}$. 
Proof: See Appendix 5.3.

It follows from these Lemmas that $\lim _{\sigma_{1} \rightarrow 0}\left(\alpha^{\sigma_{1}}, s^{\sigma_{1}}, \underline{\alpha}^{\sigma_{1}}, b^{\sigma_{1}}, \varepsilon_{r k}^{\sigma_{1}}, \underline{b}^{\sigma_{1}}, \underline{\varepsilon}_{r k}^{\sigma_{1}}\right)=$ $\left(\alpha^{0}, s^{0}, \underline{\alpha}^{0}, b^{0}, \varepsilon_{r k}^{0}, \underline{b}^{0}, \underline{\varepsilon}_{r k}^{0}\right)$. We have then proved:

Proposition 3. Let the production functions be given by (15). If $\kappa^{*} \in$ $(1 / 2,1)$, there is $\bar{\sigma}_{1}>0$ such that for all $\sigma_{1} \in\left[0, \bar{\sigma}_{1}\right)$ there exist $\bar{\eta}^{\sigma_{1}} \in(0,1)$, $\underline{\varsigma}^{\sigma_{1}}>0, \underline{\chi}^{\sigma_{1}} \in(0,1), \bar{\chi}^{\sigma_{1}} \in(0,1), \underline{\gamma}^{\sigma_{1}}>1$ and $\bar{\gamma}^{\sigma_{1}}>\underline{\gamma}^{\sigma_{1}}$ such that when $\bar{\eta}<\bar{\eta}^{\sigma_{1}}, \bar{\varsigma}>\underline{\varsigma}^{\sigma_{1}}$ and $\chi \in\left(\underline{\chi}^{\sigma_{1}}, \bar{\chi}^{\sigma_{1}}\right)$, the NSS is locally indeterminate for any $\gamma \in\left(\underline{\gamma}^{\sigma_{1}}, \bar{\gamma}^{\sigma_{1}}\right)$.

Theorem 1 and Proposition 3 show that in a two-sector OLG economy, the existence of local indeterminacy under dynamic efficiency is compatible with positive capital-labor substitution in each sector. Following Reichlin [9], our conclusion suggests that the Pareto optimal steady state can be considered as a target of the policymaker. Indeed the introduction of a public policy based on taxes and transfers could at the same time rule out business-cycle fluctuations in the economy and drive the equilibrium to the optimal steady state which provides an equal level of utility to all generations.

Let us prove now along the line of Reichlin [9] that such a policy exists under the assumption that agents and public authority do not make forecasting mistakes. Assume that the public authority buys goods, levies taxes and makes transfers. Let $g_{t}$ be the flow of consumption goods which is bought, $\tau_{t}^{y}<(>) 0$ the taxes (transfers) on the income of the young and $\tau_{t}^{o}<(>) 0$ the taxes (transfers) on the income of the old. We assume a balanced budget rule, i.e. $g_{t}+\tau_{t}^{y}+\tau_{t}^{o}=0$. The agent's first and second period budget constraints become $w_{t}+\tau_{t}^{y}=c_{t}+\phi_{t}$ and $R_{t+1} \phi_{t}+\tau_{t+1}^{o}=d_{t+1}$. From utility maximization, we get the optimal saving

$$
\phi_{t}=\frac{B\left(\delta R_{t+1} / B\right)^{\gamma}\left(w_{t}+\tau_{t}^{y}\right)-\tau_{t+1}^{o}}{R_{t+1}+B\left(\delta R_{t+1} / B\right)^{\gamma}}
$$

and a perfect-foresight competitive equilibrium satisfies

$$
(1+n) \kappa_{t+1} p_{t}=\frac{B\left(\delta R_{t+1} / B\right)^{\gamma}\left(w_{t}+\tau_{t}^{y}\right)-\tau_{t+1}^{o}}{R_{t+1}+B\left(\delta R_{t+1} / B\right)^{\gamma}}
$$

Now consider the expression of the normalization constant as given in Proposition 1 evaluated at some point $\kappa \in(0, \bar{\kappa})$, namely $B=B(\kappa)$ such that:

$$
B(\kappa)=\frac{r(\kappa)}{p(\kappa)}\left(\frac{(1+n) \kappa p(\kappa)}{\delta^{\gamma}[w(\kappa)-(1+n) \kappa p(\kappa)]}\right)^{\frac{1}{1-\gamma}}
$$

with $r(\kappa)=T_{1}(\kappa,(1+n) \kappa, 1), p(\kappa)=-T_{2}(\kappa,(1+n) \kappa, 1)$ and $w(\kappa)=$ $T_{3}(\kappa,(1+n) \kappa, 1)$. Let 


$$
\begin{aligned}
\hat{\tau}_{t+1}^{o} & =(1+n)\left\{\kappa p(\kappa)\left[R(\kappa)+B(\kappa)(\delta R(\kappa) / B(\kappa))^{\gamma}\right]\left(\frac{R_{t+1}}{R(\kappa)}\right)^{\gamma}\right. \\
& \left.-\kappa_{t+1} p_{t}\left[R_{t+1}+B(\kappa)\left(\delta R_{t+1} / B(\kappa)\right)^{\gamma}\right]\right\} \\
\hat{\tau}_{t}^{y} & =w(\kappa)-w_{t}
\end{aligned}
$$

with $R(\kappa)=r(\kappa) / p(\kappa)$. Plugging $\tau_{t}^{y}=\hat{\tau}_{t}^{y}, \tau_{t+1}^{o}=\hat{\tau}_{t+1}^{o}$ and $\tau_{t+1}^{y}=\tau_{t+2}^{o}=0$ into (17) gives

$$
(1+n) \kappa p(\kappa)=\frac{(\delta R(\kappa) / B(\kappa))^{\gamma} B(\kappa) w(\kappa)}{R(\kappa)+(\delta R(\kappa) / B(\kappa))^{\gamma}}
$$

As shown in Proposition 1, there exists a solution $\kappa=\kappa^{*}$ which corresponds to the normalized stationary Pareto optimal perfect-foresight competitive equilibrium. It follows that if agents believe in this announced policy rule, they will expect the optimal NSS allocation to hold in the future. This expectation in turn drives the sytem to the NSS and keep it there forever. Note however that contrary to Riechlin [9], when local indeterminacy holds, the NSS is not the unique stationary solution of equation (19). Indeed, as mentioned in Remark 1, a second steady state generically exists through a transcritical bifuration occurring at $\gamma$ and is locally unstable for $\gamma>\gamma$. It follows that the stabilization policy $(18)$ has to be parameterized by the right NSS in order to jump on the optimal stationary allocation.

\section{$5 \quad$ Appendix}

\subsection{Proof of Proposition 1}

Consider the set $\mathcal{K}$ as defined by (8) and the expression of $\alpha(R / B)$ as given by $(7) . \kappa^{*} \in(0, \bar{\kappa})$ is a solution of $(10)$ if

$$
\frac{1}{1+\delta^{\gamma}\left(-\frac{T_{1}\left(\kappa^{*},(1+n) \kappa^{*}, 1\right)}{T_{2}\left(\kappa^{*},(1+n) \kappa^{*}, 1\right) B}\right)^{\gamma-1}}=1+(1+n) \frac{\kappa^{*} T_{2}\left(\kappa^{*},(1+n) \kappa^{*}, 1\right)}{T_{3}\left(\kappa^{*},(1+n) \kappa^{*}, 1\right)} \in(0,1)
$$

Then there exists a unique positive value of $B$ solution of (20) given by

$$
B\left(\kappa^{*}\right)=-\frac{T_{1}\left(\kappa^{*},(1+n) \kappa^{*}, 1\right)}{T_{2}\left(\kappa^{*},(1+n) \kappa^{*}, 1\right)}\left(\frac{-(1+n) \kappa^{*} T_{2}\left(\kappa^{*},(1+n) \kappa^{*}, 1\right)}{\left[T_{3}\left(\kappa^{*},(1+n) \kappa^{*}, 1\right)+(1+n) \kappa^{*} T_{2}\left(\kappa^{*},(1+n) \kappa^{*}, 1\right)\right] \delta^{\gamma}}\right)^{\frac{1}{1-\gamma}}
$$

and $\kappa^{*}$ is a steady state if and only if $B=B\left(\kappa^{*}\right)$.

\subsection{Proof of Theorem 1}

From (7), we get

$$
\alpha^{\prime}(R / B)=(1-\gamma) \alpha(R / B)(1-\alpha(R / B)) B / R
$$


Under Assumptions 1 and 2, we get from the first order conditions of program (3)

$T_{12}=-T_{11} b<0, T_{22}=T_{11} b^{2}<0, T_{31}=-T_{11} a>0, T_{32}=T_{11} a b>0$

with $a \equiv k^{0} / l^{0}>0, b$ as defined by (12) and $T_{11}<0$. Considering (13) together with $(22), T_{1} \kappa^{*} / T_{3}=s /(1-s),-T_{1} / T_{2}=R^{*}=s /(1-\alpha)(1-s)$ and the fact that homogeneity of $T(k, y, \ell)$ implies $a=[1-(1+n) b] \kappa^{*}$, total differenciation of (9) using (4), (5) and (21) evaluated at the NSS gives the characteristic polynomial $\mathcal{P}(\lambda)=\lambda^{2}-\lambda \mathcal{T}(\gamma)+\mathcal{D}(\gamma)$ with

$$
\mathcal{D}(\gamma)=\frac{s[(1+n) b \alpha(\gamma-1)+1-\alpha+\alpha(1+n) b]}{(1+n) b(1-\alpha)(1-s) \alpha(\gamma-1)}, \quad \mathcal{T}(\gamma)=\frac{1}{(1+n) b \varepsilon_{r k} \alpha(\gamma-1)}+\frac{1+\mathcal{D}(1+n)^{2} b^{2}}{(1+n) b}
$$

When $B=B\left(\kappa^{*}\right)$, the NSS, $\alpha, s$ and $\varepsilon_{r k}$ remain constant for any $\gamma \neq 1$. We then study the variations of $\mathcal{T}(\gamma)$ and $\mathcal{D}(\gamma)$ in the $(\mathcal{T}, \mathcal{D})$ plane as $\gamma$ varies within $(1,+\infty)$. Solving $\mathcal{T}(\gamma)$ and $\mathcal{D}(\gamma)$ with respect to $\alpha(\gamma-1)$ yields to

$$
\begin{aligned}
\mathcal{D}=\Delta(\mathcal{T}) & =\frac{\varepsilon_{r k} s[1-\alpha+\alpha(1+n) b] \mathcal{T}}{(1-\alpha)(1-s)+\varepsilon_{r k} s(1+n) b[1-\alpha+\alpha(1+n) b]} \\
& -\frac{\varepsilon_{r k} s[1-\alpha+\alpha(1+n) b]-s(1+n) b}{(1+n) b\left[(1-\alpha)(1-s)+\varepsilon_{r k} s(1+n) b(1-\alpha+\alpha(1+n) b)\right]}
\end{aligned}
$$

and allows to use the methodology introduced by Grandmont et al. [7]. As $\gamma$ spans the interval $(1,+\infty), \mathcal{T}(\gamma)$ and $\mathcal{D}(\gamma)$ vary linearly along the line $\Delta(\mathcal{T})$. The fundamental properties of $\Delta(\mathcal{T})$ depend on its extremities. The starting point of the pair $(\mathcal{T}(\gamma), \mathcal{D}(\gamma))$ is obtained when $\gamma=+\infty$ :

$$
\mathcal{D}(+\infty)=\mathcal{D}_{\infty}=\frac{s}{(1-\alpha)(1-s)}, \mathcal{T}(+\infty)=\mathcal{T}_{\infty}=\frac{(1-\alpha)(1-s)+(1+n)^{2} b^{2} s}{(1+n) b(1-\alpha)(1-s)}
$$

while the end point is obtained when $\gamma$ converges to 1 from above. Let $\bar{b}=-1 /(1+n), \underline{b}=-(1-\alpha) /(1+n) \alpha$ and $\hat{b}=-(1-\alpha)(1-s) /(1+n) s$. As $\lim _{\gamma \rightarrow 1^{+}} \mathcal{D}(\gamma)=\lim _{\gamma \rightarrow 1^{+}} \mathcal{T}(\gamma)=\infty, \Delta(\mathcal{T})$ is a half-line starting from $\left(\mathcal{T}_{\infty}, \mathcal{D}_{\infty}\right)$ and pointing downwards or upwards depending on the sign of

$$
\mathcal{D}^{\prime}(\gamma)=\frac{s(\underline{b}-b)}{b(1-\alpha)(1-s)(\gamma-1)^{2}}
$$

Under Assumptions 1-2, we get from (23) that $\mathcal{D}_{\infty}=R^{*} /(1+n)>1$. Local indeterminacy then requires that $\mathcal{D}^{\prime}(\gamma)>0$, i.e. $b \in\left(b_{1}, 0\right)$ as shown by (24). But in this case, $\mathcal{D}=1$ implies $\mathcal{T}<-2$. Indeed, solving $\mathcal{D}=1$ gives

$$
\alpha(1-\gamma)=\frac{\alpha s(b-\underline{b})}{b(1-s)(\alpha-\underline{\alpha})}
$$

which can be satisfied if and only if $b \in(\underline{b}, 0)$. Moreover, substituting $\mathcal{D}=1$ into the expression of $\mathcal{T}$ allows to get

$$
\mathcal{T}+2=\frac{1}{(1+n) b \varepsilon_{r k} \alpha(\gamma-1)}+\frac{[1+(1+n) b]^{2}}{(1+n) b}<0
$$

Therefore, when $b \in(\underline{b}, 0)$, the only possibility to get local indeterminacy is that the $\Delta$-half-line crosses the interior of segment $[A C]$ as follows 


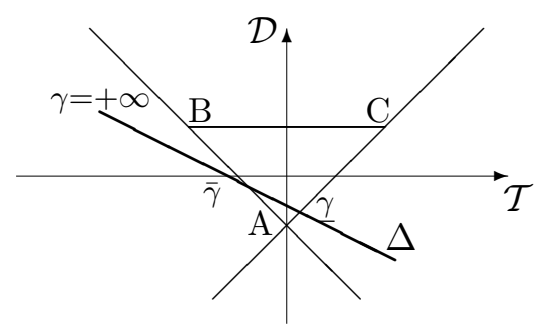

Figure 1: Local indeterminacy with dynamic efficiency.

This requires that $1+\mathcal{T}_{\infty}+\mathcal{D}_{\infty}=(\bar{b}-b)(b-\hat{b}) / b \hat{b}<0 \Leftrightarrow b \in(-\infty, \bar{b}) \cup(\hat{b}, 0)$, and that $\mathcal{D}=-1$ implies $\mathcal{T}>0$. Solving $\mathcal{D}=-1$ gives

$$
\alpha(1-\gamma)=\frac{\alpha s(b-\underline{b})}{b[1-\alpha(1-s)]}
$$

which holds if and only if $b \in(\underline{b}, 0)$. Substituting $\mathcal{D}=-1$ into $\mathcal{T}$ gives

$$
\mathcal{T}=\frac{\varepsilon_{r k} \alpha s(b-\underline{b})(1+n)^{2}\left(\bar{b}^{2}-b^{2}\right)-b[1-\alpha(1-s)]}{(1+n) b \varepsilon_{r k} \alpha s(b-\underline{b})}
$$

Noting that $\underline{b}<\bar{b} \Leftrightarrow \alpha<1 / 2$ and $\underline{\alpha}<1 / 2 \Leftrightarrow s>1 / 3$, we derive from (28) that $\Delta(\mathcal{T})=-1$ implies $\mathcal{T}>0$ if and only if $s \in(1 / 3,1 / 2)$, $\alpha \in(\underline{\alpha}, 1 / 2), b \in(\underline{b}, \bar{b})$ and $\varepsilon_{r k}>\underline{\varepsilon}_{r k}$ with

$$
\underline{\varepsilon}_{r k}=\frac{b[1-\alpha(1-s)]}{(1+n)^{2}\left(b_{0}^{2}-b^{2}\right) \alpha s\left(b-b_{1}\right)}>0
$$

The result follows.

\subsection{Proof of Lemma 2}

We use the same kind of argument as Nishimura and Yano ([8], Lemma 1, p. $229)$. Define the set $E\left(k, y, \ell, \sigma_{1}\right)=\left\{\left(k^{0}, l^{0}\right) \geq 0 \mid y \leq f^{1}\left(k-k^{0}, \ell-l^{0}\right)\right\}$. Then $T^{\sigma_{1}}(k, y, \ell)=\max f^{0}\left(k^{0}, l^{0}\right)$ s.t. $\left(k^{0}, l^{0}\right) \in E\left(k, y, \ell, \sigma_{1}\right)$. Since $E\left(k, y, \ell, \sigma_{1}\right)$ is lower-semi-continuous in $\left(k, y, \ell, \sigma_{1}\right), T^{\sigma_{1}}(k, y, \ell)$ is continuous. It follows also that the functions $\left(k_{\sigma_{1}}^{0}(k, y, \ell), l_{\sigma_{1}}^{0}(k, y, \ell)\right)=\operatorname{argmax} f^{0}\left(k^{0}, l^{0}\right)$ s.t. $\left(k^{0}, l^{0}\right) \in E\left(k, y, \ell, \sigma_{1}\right)$ are continuous. Moreover, as $\lim _{\rho \rightarrow+\infty} f^{1}\left(k^{1}, l^{1}\right)=$ $\min \left\{k^{1} / \eta, l^{1}\right\}$, we can find a sub-sequence $\sigma_{1 i}$ such that when $\sigma_{1 i} \rightarrow 0$, $\left(k_{\sigma_{1}}^{0}(k, y, \ell), l_{\sigma_{1}}^{0}(k, y, \ell)\right) \rightarrow(k-\eta y, \ell-y)$ for any $(k, y, \ell) \in \tilde{\mathcal{K}}$. Therefore, the uniform convergence follows and $\lim _{\sigma_{1} \rightarrow 0} T^{\sigma_{1}}(k, y, \ell)=T^{0}(k, y, \ell)$. 


\section{References}

[1] Benhabib, J. and K. Nishimura (1981): "Stability of Equilibrium in Dynamic Models of Capital Theory," International Economic Review 22, 275-293.

[2] Cazzavillan, G. and P. Pintus (2006): "Endogenous Business Cycles and Dynamic Inefficiency," International Journal of Economic Theory 2, 279-294.

[3] Cazzavillan, G. and P. Pintus (2007): "Dynamic Inefficiency in an Overlapping Generations Economy with Production," Journal of Economic Theory 137, 754-759.

[4] Drugeon, J.P. (2004): "On Consumptions, Inputs and Outputs Substitutabilities and the Evanescence of Optimal Cycles," Journal of Difference Equations and Applications 10, 473-487.

[5] Drugeon, J.P., C. Nourry and A. Venditti (2006): "Does Dynamic Efficiency Rule Out Expectation-Driven Fluctuations ?," GREQAM Working Paper.

[6] Galor, O. (1992): "A Two-Sector Overlapping-Generations Model: a Global Characterization of the Dynamical System," Econometrica 60, 1351-1386.

[7] Grandmont, J.M., P. Pintus and R. De Vilder (1998): "Capital-Labor Substitution and Competitive Nonlinear Endogenous Business Cycles," Journal of Economic Theory 80, 14-59.

[8] Nishimura, K. and M. Yano (1998): "Interior Optimal Chaos with Arbitrarily Low Discount Rates," Japanese Economic Review 49, 223233.

[9] Reichlin, P. (1986): "Equilibrium Cycles in an Overlapping Generations Economy with Production," Journal of Economic Theory 40, $89-102$.

[10] Reichlin, P. (1992): "Endogenous Fluctuations in a Two-Sector Overlapping Generations Economy," in Benhabib J. (ed.), Cyles and Chaos in Economic Equilibrium, Princeton University Press, Princeton. 